DOI: $10.35643 /$ Info.25.2.1

Artículo original

\title{
Socioterminología: orígenes, objeto y aplicaciones
}

\author{
Socioterminology: origins, object and application
}

Socioterminologia: origens, objeto e aplicações

\begin{abstract}
Sileymane Diallo ${ }^{\mathrm{a}}$
${ }^{a}$ Doctorando en socioterminología. Laboratoire RSD. Section de Langues Étrangères Appliquées UFR de Lettres et Sciences Humaines. Université Gaston Berger, Senegal. Correo electrónico: diallo.sileymane@ugb.edu.sn. ORCID: 0000-0002-9531-6010
\end{abstract}

\section{Resumen}

La socioterminología, concepto utilizado por primera vez por Jean-Claude Boulanger en 1981, es un enfoque que nació de los límites percibidos en lo que concierne a la terminología dominante de tipo wüsteriano. Por consiguiente, se fundó y se ha desarrollado en respuesta a una demanda social en cuanto al vocabulario o al discurso especializado. Desde su creación hasta la fecha de hoy, muchos estudios se han enmarcado dentro de este planeamiento, a pesar de que su uso es limitado en varias áreas del mundo. Partiendo de esta observación, hemos decidido llevar a cabo un estudio cuyo objetivo es participar en la divulgación de esta perspectiva de investigación. También, hemos querido describir el estado de la cuestión de los estudios que han investigado el carácter operativo de los conceptos elaborados y que han aplicado los métodos preconizados por la socioterminología.

Palabras clave: TERMINOLOGÍA; TERMINOLOGÍA WÜSTERIANA; SOCIOTERMINOLOGÍA; DISCURSO ESPECIALIZADO; DIVULGACIÓN.

\footnotetext{
Abstract

Socioterminology, a concept first used by Jean-Claude Boulanger in 1981, is an approach that was born out of the perceived limits of the dominant Wüsterian-type terminology. It was therefore launched and developed in response to a social demand for specialized vocabulary or discourse. From its inception to the present day, many studies have been part of this approach, even though its use is limited in several areas of the world. On the basis of this state of affairs, we have decided to carry out a study whose purpose is to participate in the popularization of this research perspective. We have likewise aimed at describing the state of the art regarding the studies that have ascertained the operational nature of the concepts elaborated and that have applied the methods advocated by Socioterminology.

Keywords: TERMINOLOGY; WÜSTERIAN-TYPE TERMINOLOGY; SOCIOTERMINOLOGY; LSP; POPULARIZATION.
} 


\section{Resumo}

A socioterminologia, um conceito usado pela primeira vez por Jean-Claude Boulanger em 1981, é uma abordagem que nasceu dos limites percebidos da terminologia do tipo Wüsteriano dominante. Consequentemente, foi fundado e desenvolvido em resposta a uma demanda da sociedade por vocabulário ou discurso especializado. Desde a sua criação até o momento, muitos estudos têm se enquadrado nesse planejamento, apesar de seu uso ser limitado em várias regiões do mundo. A partir dessa constatação, decidimos realizar um estudo cujo objetivo é participar da divulgação dessa perspectiva de pesquisa. Além disso, queríamos descrever o estado da arte dos estudos que investigaram a natureza operativa dos conceitos elaborados e que aplicaram os métodos preconizados pela socioterminologia.

Palavras-chave: TERMINOLOGIA; TERMINOLOGIA WÜSTERIANA; SOCIOTERMINOLOGIA; DISCURSO ESPECIALIZADO; DIVULGAÇÃO.

Fecha de recibido: $\quad$ 14/06/2020

Fecha de aceptado: $\quad 13 / 08 / 2020$

\section{Introducción}

Unos cuarenta años atrás, se utilizó por primera vez, del puño y letra de JeanClaude Boulanger, el término socioterminología, en su reseña de una obra de Jean-Claude Corbeil. En aquel entonces, era un mero hápax. Fue a partir de 1990 cuando se empezó a utilizar el término de manera frecuente y a desarrollar la socioterminología como perspectiva de investigación. Unos treinta años después, muchos trabajos de índole científica son parte de este enfoque. Estos trabajos se extienden por el continente boreal, incluyendo Quebec, por parte de los mencionados autores y, también, por lingüistas como Pierre Auger y Christiane Loubier, entre otros. Asimismo, en la actualidad, se están desarrollando en Europa, en países como Francia (véase el laboratorio de Ruan), Noruega (Johan Myking), así como en Finlandia (Yves Gambier) y, en mucho menor medida, en Sudamérica (Argentina, Brasil y Uruguay). Sin embargo, excepto en Francia y en Quebec, el uso e, incluso, el éxito de este planteamiento en otras áreas es limitado, pese a que algunos estudios se han interesado por la socioterminología o se están suscribiendo a esta perspectiva de investigación. En ciertas zonas, tales como África, el concepto está aún poco desarrollado y es prácticamente desconocido en algunos países hispanohablantes. Esto se debe, en cierto modo, a que las orientaciones socioterminológicas no se han difundido allí de manera extensa. Por 
una parte, el objetivo de este trabajo es, teniendo en cuenta las afirmaciones anteriores, impulsar la divulgación de dicho enfoque en países cuyas lenguas son menos conocidas o estudiadas. Por otra parte, presentar el estado de la cuestión sobre la implementación de los métodos preconizados por el planteamiento socioterminológico en estudios realizados, casi todos en Francia, de los cuales la mayoría versan sobre lenguas que tienen menos trabajos terminológicos y terminográficos. Para ello, en primer lugar, este trabajo procura hacer un historial de la actividad socioterminológica, profundizando en los factores que han contribuido a la creación y a la evolución del concepto. En segundo lugar, se ocupa del objeto de la socioterminología, haciendo hincapié en su orientación, finalidad y método de análisis. En tercer lugar, analiza la implementación de los métodos preconizados por la socioterminología en distintos estudios científicos y se dan a conocer los resultados a los que se ha llegado a través de dichos estudios.

\section{Historial de la socioterminología}

\subsection{Contexto de nacimiento de la perspectiva de investigación}

Hasta la creación de la Escuela Vienesa de Terminología (EVT), solía haber una especie de desinterés recíproco entre lingüistas y terminólogos científicos en cuanto al objeto de estudio de cada uno. En efecto, la mayoría de los lingüistas no se interesaban por el vocabulario especializado. Tampoco los científicos usaban los conocimientos adquiridos en el ámbito de la lingüística. Las cosas cambiaron como resultado de los trabajos del pionero en la teoría moderna de la terminología, Eugene Wüster (1898-1977). Fue a partir de aquel entonces cuando comenzaron los trabajos en lingüística sobre los vocabularios científico y técnico. En 1979, Felbert, Lang y Wersig publicaron un libro titulado La terminología como ciencia lingüística aplicada. Dicho libro, editado en memoria del fundador de la teoría moderna de la terminología, sancionó la pertenencia de la terminología a la lingüística aplicada. La terminología se convirtió, así, en una rama de la lingüística experimental. Mientras tanto, surgieron dos corrientes de investigación lingüística sobre el discurso especializado. Por un lado, afloraron las reflexiones didácticas realizadas en el momento de la constitución de lo que se llamó el francés funcional (1972-1973); por otro lado, los planteamientos más 
teóricos desarrollados desde una óptica estructural (Delavigne, 2001). Seguidamente, el análisis lingüístico condujo a un discurso especializado, más específicamente, hacia el análisis del discurso. En este caso también, como lo señala Delavigne (2001), destacaron dos orientaciones. Una de ellas es de carácter terminológico: la socioterminología, enfoque que se le da al presente artículo.

La socioterminología nació de la crisis (de crecimiento, métodos, autoridad e identidad) de la terminología dominante de tipo wüsteriano. Esta terminología se centraba particularmente en tres aspectos: el estudio de las nociones, el estudio de las denominaciones y la normalización. No daba cuenta del funcionamiento de las nociones ni de lo social de los términos, ni usaba, tampoco, un planteamiento explicativo. Esto llevó a algunos especialistas a cuestionar las orientaciones de la terminología dominante de tipo wüsteriano y a militar a favor de un acercamiento social de la terminología. De ahí surgió el concepto socioterminología, el cual es una combinación de dos vocablos: sociolingüística y terminología.

En el movimiento que condujo a la reforma de las teorías y prácticas, François Gaudin (2005) identifica cuatro fuentes principales: sociolingüística teórica, sociolingüística de campo, lingüística general y lingüística de corpus. Según dicho autor, la sociolingüística teórica permitió retomar las concepciones del discurso desde una perspectiva derivada de la sociolingüística de covariación y de los trabajos sobre las interacciones verbales. En lo que atañe a la sociolingüística de campo, propició mejoras en materia de política lingüística cuando la lingüística general permitió replantear el estatus del término, en tanto que signo, y cuestionar el vínculo entre términos y referentes. La lingüística de corpus, por su parte, impuso una reforma de los métodos y de las concepciones para el desarrollo de la gestión informatizada de los escritos y la aparición de nuevas herramientas lingüísticas.

\subsection{La socioterminología como «hija de la sociolingüística» y la evolución del enfoque}

Fue mediante una postura resueltamente crítica con respecto a los postulados de la terminología wüsteriana, así como mediante la integración de los conceptos sociolingüísticos en el estudio del discurso especializado, «como surgió la socioterminología» (Delavigne, 2001, p. 204). Dicho de otro modo, la 
socioterminología nació del cuestionamiento de las herramientas conceptuales de la terminología originada en la escuela de Wüster, combinada con un deseo de ocuparse de lo social en el uso de los términos. La socioterminología, tal y como la concibe Gaudin (2003), es un campo en el que se encuentran nuevas cuestiones terminológicas y problemáticas alentadas por la sociolinguiística. Se interesa por los usos sociales de los términos, el estudio de su aparición, circulación e implantación, lo cual hace de ella una perspectiva de investigación que está a caballo entre la sociolingüística y la terminología. Además, explota los conocimientos conceptuales y metodológicos de la sociolingüística para aplicarlos al discurso especializado. Esto llevó a François Gaudin a afirmar que la socioterminología es «la hija de la sociolingüística» (Gaudin, 1990, p. 177). Sin embargo, cabe señalar que su significado y su uso como perspectiva de investigación tardaron en generalizarse.

Como ya hemos dicho, el término socioterminología apareció por primera vez en 1981 de la mano de Jean-Claude Boulanger, en la reseña de una obra de JeanClaude Corbeil. Enseguida, lo utilizaron, sucesivamente, Pierre Lerat y Monique Slodzian, pero siguió siendo un hápax. Su contenido comenzó a construirse de manera pragmática a partir de 1986, después de una ponencia de Yves Gambier en el coloquio sobre la fertilización en las lenguas romances cuyo tema era: «Problemas terminológicos de la lluvia ácida: por una socioterminología» (Gambier, 1987). A pesar de que, por su contenido pragmático, esa comunicación se consideró pionera, no fue hasta 1990 cuando se estableció, finalmente, la socioterminología como disciplina. Ello resultó posible gracias a los trabajos realizados en la Universidad de Ruan bajo la coordinación de Louis Guespin. Aunque la elaboración del contenido semántico adelantó el uso del significante (socioterminología), Valérie Delavigne piensa que se debe acreditar a Louis Guilbert y a Alain Rey el haber vinculado la terminología con la sociolingüística (Delavigne, 2001).

Por último, cabe recordar que algunos estudios terminológicos comenzaron a realizarse en el ámbito de la sociolingüística desde principios de 1970. Asimismo, la contribución de la sociolingüística a la terminología se hacía notar desde el decenio de 1980, particularmente en Quebec, con, entre otras obras, las de Monica Heller et al. Por eso, Jean-Claude Boulanger, quien utilizó la palabra por primera 
vez, dijo: «Dans la Belle Province, la socioterminologie vit depuis longtemps en filigrane» (Boulanger, 1995, p. 15). En otras palabras, las orientaciones socioterminológicas gozan de influencia en Quebec desde hace mucho tiempo. En resumidas cuentas, como lo subraya Rodolfo Alpízar (citado por Borbujo, 2001), la socioterminología resultó de la inadecuación de la teoría y la práctica terminológicas wüsterianas a la realidad de la lengua. Por lo tanto, se preconizó un planteamiento más sociolingüístico de la terminología, que iba a posibilitar, sobre todo, una comunicación científica «más fluida y eficiente» (Borbujo, 2001). A continuación, veremos el objeto de la socioterminología, su orientación, finalidad y método de análisis.

\section{Objeto, orientación, finalidad y método de análisis de la socioterminología}

A semejanza de cualquier disciplina o perspectiva de investigación, la socioterminología tiene un objeto, una orientación, finalidad y método de análisis. Estos constituyen el objeto de las líneas siguientes.

\subsection{Objeto de estudio y método de análisis de la socioterminología}

La socioterminología se ocupa de los términos, siendo la circulación social de estos últimos su objeto de estudio. La difusión terminológica se puede estudiar desde una óptica sincrónica o diacrónica, lo cual engloba el análisis y la modelización de significados y conceptualizaciones (Gaudin, 1990). La circulación de los términos se examina desde la perspectiva de la diversidad de sus usos sociales, lo cual abarca, según Gaudin, tanto el estudio de las condiciones de circulación como la apropiación de los términos, considerándolos no ya meras denominaciones de conceptos, sino más bien signos lingüísticos (Gaudin, 1990). Los signos lingüísticos se actualizan mediante discursos; estos pueden implementarse en discursos en lengua común o en lenguas especializadas. En el primer caso se les llama palabras, mientras que en el segundo caso gozan del estatus de términos. Por ende, podemos decir que es en los discursos especializados donde se encuentran los signos lingüísticos que constituirán, a continuación, el objeto de la socioterminología. Por eso, Delavigne opina que el ámbito de la socioterminología radica en la realidad de los discursos 
especializados en los que se insertan los términos, los cuales se implementan a lo largo de interacciones específicas (Delavigne, 1995).

La recopilación de términos se hace por medio de un enfoque llamado socioterminografía. El enfoque socioterminográfico consiste en recoger los signos lingüísticos en su contexto discursivo. Este enfoque «fundado en corpus y procedimientos de entrevista, permite describir las prácticas lingüísticas reales y analizar en profundidad las manifestaciones terminológicas en situaciones interactivas y discursivas» (Delavigne, 1994, p. 2). En este planeamiento, se pueden tomar en cuenta tanto las fuentes escritas como las orales, aunque, hoy en día, las últimas, a saber, las orales, continúan siendo apenas estudiadas. Una vez identificados los signos lingüísticos y el/los discurso(s) especializado(s) en el/los que se enmarcan, hay dos tipos de análisis que se le ofrece al investigador dedicado al enfoque socioterminológico. Por una parte, están los análisis relacionados con los términos $\mathrm{y}$, por otra, los discursivos. En este último caso tenemos la perspectiva metodológica de la semántica lingüística, la que estudia el uso de la lengua, en tanto que hecho de comunicación e interacción en sus contextos cognitivos y sociales: el análisis del discurso. En cuanto a los términos y entre los diferentes tipos de análisis al respecto, se preconiza y privilegia la semasiología.

Tras haber estudiado el objeto de la socioterminología, el socioterminólogo se ocupará de identificar los problemas al respecto. Estos pueden ser relativos a cómo se han elaborado los términos, a su intercomprensibilidad, a sus usos y variaciones en los discursos, a los procedimientos metadiscursivos utilizados, a las difusiones sociales de los términos y de los discursos especializados, etcétera. De ahí, es de su incumbencia proponer soluciones que posibiliten la intercomprensibilidad, la univocidad, la erradicación de ambigüedades en las comunicaciones especializadas, entre otras. En resumidas cuentas, las orientaciones socioterminológicas pretenden reducir el desorden terminológico, a falta de erradicarlo, y permitir la intercomprensión en cuanto a las comunicaciones especializadas. Para ello, los socioterminólogos colocan a los locutores en el centro de sus estudios. De ellos proceden los términos; de los discursos que producen se derivan los términos. Sobre los usos sociales que hacen de los términos versan los análisis, sobre ellos se prueban los términos y con ellos 
se negocian las soluciones que se propondrán con fines de intercomprensibilidad, univocidad e intercomprensión en los discursos especializados. Esta orientación guarda relación con la estrategia recomendada por el enfoque socioterminológico, a saber, la glotopolítica. Es este un concepto desarrollado en los años 1980 por Louis Guespin y Jean-Baptiste Marcellesi. Dichos autores opinan que cualquier medida, en relación con lo social de los términos o de los discursos, entra en el marco de la glotopolítica (Guespin y Marcellesi, 1986). Habiendo quedado claro que se enfocan los términos y los discursos según la vara de medir de su contenido social, las decisiones socioterminológicas se subscriben, sin lugar a dudas, a la perspectiva glotopolítica. En efecto, tal y como apuntan Guespin y Marcellesi (1986), «desde el punto de vista del lingüista, cualquier decisión que modifica las relaciones sociales es una decisión glotopolítica» (p. 15) . En lo que sigue, se estudiarán los objetivos de la socioterminología, haciendo hincapié en lo que los diferencia de los de la terminología wüsteriana y en lo común que tienen estas dos orientaciones.

\subsection{Socioterminología vs terminología wüsteriana}

Louis-Jean Rousseau (1993) considera la socioterminología como un planteamiento de carácter situacional, el que se dedica a las condiciones en las cuales se producen los textos científicos y técnicos y a las circunstancias que causan la variación terminológica, sea geolectal o sociolectal. En otras palabras, la observación de los vocabularios especializados en corpus socialmente diversos es el principal objeto de estudio de la socioterminología. A la vez que observa y estudia los términos de los ámbitos especializados en su contexto discursivo, la socioterminología cuestiona las realidades lingüísticas, lo cual la demarca neta y claramente de la terminología, tal y como se la entiende por la teoría general (TGT) concebida por el fundador de la EVT, Eugene Wüster. En efecto, son muy distintos los objetivos que persigue la TGT y la socioterminología, cada una en lo que le concierne. Así, los tres objetivos principales en los que la terminología tiene las miras puestas y que la distinguen claramente de la socioterminología son los siguientes: la voluntad de normalización a ultranza, la erradicación de la polisemia y la preocupación por la univocidad desde el prisma de la perspectiva. La socioterminología, en cambio, pretende, esencialmente y antes que nada, describir 
y entender la circulación de los términos y luego captar su funcionamiento. Por fin, mientras que la terminología privilegia el enfoque onomasiológico, la socioterminología, por su parte, favorece el enfoque opósito, a saber, la semasiología. Sin embargo, por lo distinto que puedan ser los objetivos que persiguen ambas orientaciones, la socioterminología no puede desvincularse completamente de la terminología. Ese es el punto de vista de los investigadores, para quienes la socioterminología es parte de la terminología como área de conocimiento. De los autores que sostienen que la socioterminología sigue siendo una rama de la terminología como área de conocimiento, podemos mencionar a Mario Barité. En su diccionario electrónico define la socioterminología de la siguiente manera:

Socioterminología. // 1. Rama de la Terminología que se ocupa del análisis de los términos (surgimiento, formación, consolidación e interrelaciones), considerándolos desde una perspectiva lingüística en la interacción social. // 2. Disciplina eminentemente práctica del trabajo terminológico, que se fundamenta en el análisis de las condiciones sociales y lingüísticas de circulación de los términos (Barité, 2000).

A raíz de estas dos definiciones dadas del concepto socioterminología, se puede notar que hay una recurrencia del término (raíz) término. Es decir, no importa desde qué ángulo se entienda el concepto, siempre mantiene un vínculo con la terminología. En la primera definición, se trata de una «rama de la terminología», mientras que, en la segunda, el autor habla de «disciplina en el trabajo de la terminología». Con esto, queremos decir que las reflexiones socioterminológicas raramente pueden prescindir de lo relacionado con la terminología. Ahora bien, lo que hace falta destacar a través de dichas definiciones es, especialmente, el análisis de las condiciones sociales y lingüísticas de la circulación de los términos. Habiendo dicho esto, las reflexiones socioterminológicas permiten descubrir y desentrañar el conocimiento del funcionamiento discursivo y social de las entidades terminológicas (términos). Las reflexiones terminológicas se orientan, por su parte, a una óptica logicista y normalizadora. No están tan interesadas en las comunicaciones especializadas, las cuales constituyen la pieza clave de las reflexiones socioterminológicas. En el capítulo siguiente, se estudiarán las aplicaciones de los métodos preconizados por la socioterminología en distintos estudios científicos. 


\section{Estudios teóricos y aplicaciones}

Tal y como hemos mencionado en la introducción, hoy en día se cuenta con varios trabajos de índole científica que se han subscrito, directa o indirectamente, a la perspectiva de investigación socioterminológica. Respecto del marco teórico, se han fomentado asuntos terminológicos en estudios sociolingüísticos. Entre dichos estudios, podemos destacar, en orden cronológico, entre otros, los siguientes: Guespin y Marcellesi (1986), Pour la glottopolitique; Guespin y Laroussi (1989), Glottopolitique et standardisation terminologique; Guespin (1993), Normaliser ou standardiser; Faulstich (1999), Principes formels et fonctionnels de la variation en terminologie; Gambier (2001) Socioterminologie: une terminologie remise sur ses pied; Gaudin (2003) Socioterminologie. Une approche sociolinguistique de la terminologie.

En dichos estudios, en términos generales, los autores han tratado sobre todo de fundamentar teóricamente la socioterminología mediante la gestión social de vocabularios o terminologías. En lo que sigue, vamos a ver cómo los fundamentos teóricos de la socioterminología, sus orientaciones y los métodos que preconiza se han aplicado en estudios posteriores a los que hemos citado anteriormente. Los trabajos cuyas perspectivas y cuyos resultados vamos a abordar fueron presentados en Francia, aunque la mayoría tratan sobre lenguas poco conocidas o con pocos trabajos terminológicos.

\subsection{La sociolingüística aplicada a la terminología}

A continuación, presentamos los trabajos que tienen en cuenta las preocupaciones sociolingüísticas en el marco de la terminología, lo cual hace que se enfoquen indirectamente en el planeamiento socioterminológico.

\subsubsection{Ordenación del discurso científico y su difusión en la sociedad}

Utilizando el vocabulario atípico de la probabilidad y la estadística en el ámbito de las matemáticas, Holzem (1997) esboza un estudio socioterminológico y etiológico de la comunicación científica. En efecto, tras haber valorado las consecuencias de la evolución de las prácticas científicas de la corriente positivista, a nivel de la difusión de los conocimientos y de la lengua francesa, 
llega a proponer un modelo de ordenación del francés científico, basándose en la lista del directorio unificado de autoridad enciclopédica y alfabética de la materia. Esto se hace posible merced a un análisis de las convergencias entre la terminología y la documentación desde el punto de vista de la clasificación, lo cual lleva a la autora a trazar un camino de complementariedad entre la terminología y la documentación. Por esta razón, estamos de acuerdo con la afirmación de Gaudin (2005), respecto a que Holzem contribuye a aclarar las relaciones entre la documentación, la terminología y la sociolingüística, de modo de sentar las bases para la unificación de los métodos descriptivos.

\subsubsection{Estudio del oral en el discurso especializado}

A través de la observación participativa, Vecchi (1999) se interesa por las hablas de empresa, que propone llamar sociolecto. Habiendo considerado que el lenguaje de las empresas (o el sociolecto) es el conjunto de los procesos lingüísticos que actualizan los repertorios lingüísticos de una empresa, el autor demuestra que las herramientas teóricas de la terminología pueden apoyar la estructuración de los conceptos en marcha en una empresa hacia una terminografía evolutiva. El interés de su trabajo es doble: por un lado, se centra en lo oral, que sigue siendo muy poco estudiado en el discurso especializado; por otro, realiza una importante contribución socioterminológica al lenguaje de empresa, ámbito de la sociolingüística poco estudiado por los investigadores.

\subsubsection{Construcción de términos y sus usos en discursos}

La descripción de la terminología informática es objeto de un estudio realizado por An-Vo (2015), cuya problemática se enmarca, en particular, en la socioterminología, la lingüística de corpus y la morfología léxica. A tal fin, An-Vo se vale de los logros de la socioterminología, entre otros. Ello le permite describir conceptualmente la construcción del conocimiento de los términos informáticos utilizados en el discurso durante todos los procesos de producción, recepción y circulación social, desde sus dimensiones histórica, socioeconómica, tecnológica, coyuntural y pragmática. 


\subsubsection{Uso de las técnicas de encuesta de la sociolingüística}

En su investigación, Ngwaba (2016) utiliza las herramientas conceptuales y metodológicas de la sociolingüística para estudiar la terminología de la ginecología-obstetricia en la lengua igbo. En este sentido, emplea técnicas de encuesta de la sociolingüística para identificar y clasificar los términos de dicha área. Además, se basa en el enfoque socioterminológico para examinar la adecuación entre la terminología del igbo y la terminología internacional de los idiomas más difundidos, como el inglés y el francés. Al final de su trabajo, gracias a los métodos preconizados por la socioterminología, consigue tapar las lagunas observadas en la terminología estudiada en relación con la del inglés y del francés.

\subsection{La perspectiva socioterminológica}

A continuación figuran los trabajos de campo que se han subscrito directa y llanamente a las orientaciones socioterminológicas. Sin pretender ser exhaustivos, vamos a examinar los estudios más destacados en los cuales se han aplicado las herramientas de análisis socioterminológicas.

\subsubsection{Normalización y variación}

Al vincular la normalización (el área de la TGT) y la variación (el área de la sociolingüística), Trân (1999) contribuye en gran medida al desarrollo de la terminología vietnamita. En su obra, Trân parte del principio de que la socioterminología ya no insiste en el lenguaje regulado por expertos y normas, sino que hace hincapié en las prácticas lingüísticas. De este modo, sobre la base de las propuestas de esta perspectiva de investigación, realiza un estudio sobre la construcción del sentido del término y sobre la problemática de normalización de la terminología médica vietnamita a través de los contextos discursivos relacionados con la semántica de los términos. Esto le permite describir el funcionamiento del término médico dentro del discurso especializado.

Esta contribución socioterminológica, que culmina con el establecimiento de un diccionario bilingüe vietnamita-francés, cuestiona el valor de un término en cuanto a su uso real en la práctica sociodiscursiva. Para Trân, desde el punto de vista sociolingüístico, este diccionario tiene por objeto, ante todo, satisfacer las 
necesidades en materia de transferencia de conocimientos de todos los médicos vietnamitas o futuros médicos que intervengan en la dinámica social de la comunidad médica francófona y experimentar con un modelo de normalización terminológica mediante un enfoque sociolingüístico.

\subsubsection{Análisis de discursos de vulgarización científica}

Por medio de un análisis discursivo de la divulgación nuclear, la tesis de Delavigne (2001) constituye un extenso estudio socioterminológico dedicado al desarrollo y a la circulación social del vocabulario especializado. En este estudio, la autora se afana por determinar dicho vocabulario y establecer «criterios lingüísticos que van más allá de la intuición»(Delavigne, 2001, p. 8). Para cumplir con esto, identifica y analiza el discurso de vulgarización nuclear y disecciona sus diversos componentes léxicos para determinar si una palabra en particular es un término o un no término. En esta tesis, la autora plantea la hipótesis de que, en la circulación social del discurso sobre la energía nuclear, los términos desempeñan un papel determinado y que su uso diferenciado puede causar «efectos de sentido», los cuales pueden provocar ansiedad o reacciones disfóricas (Delavigne, 2001, p. 9). Esto la lleva a examinar la cuestión de la unidad terminológica, un concepto cuya definición resulta muy poco precisa, y a proponer herramientas teóricas y metodológicas para identificar los términos y comprender cómo funcionan en discursos de divulgación científica. Todo esto se vuelve posible gracias al carácter particular de su trabajo, que radica en la combinación de métodos de análisis de la sociolingüística, así como la utilización de las herramientas informáticas que pertenecen a la lingüística de corpus.

\subsubsection{Circulación social del discurso especializado}

Gracias a la terminología desarrollada por Sigmund Freud, con la colaboración de Jacques Lacan, y traducida al español, Gentile (2007) lleva a cabo un análisis de la circulación social del discurso psicoanalítico en este idioma. Los datos especializados que había recopilado los analiza desde la perspectiva de la implantación terminológica y las encuestas sociolingüísticas. Los resultados socioterminológicos a los que ella llega están destinados, a su parecer, a poner de relieve la pluralidad de normas que actúan en este tipo de discurso. 


\subsubsection{Transmisión del conocimiento científico}

En una tesis cuyo objetivo es construir una base de datos terminológica trilingüe que cumpla con los requisitos de especialistas y no especialistas por igual, Tajo (2013) adopta un enfoque socioterminológico y textual en el campo de la sordera. Esto le permite, entre otras cosas, arrojar luz sobre los fenómenos estudiados, como la sinonimia, la variación terminológica, la difusión de conocimientos, la metáfora y la traducción.

\subsubsection{Equipamiento terminológico y circulación social de los términos}

Trang-Nguyen, un investigador vietnamita, hace una gran aportación socioterminológica acerca de los problemas de archivo, la divulgación técnica y, por encima de todo, el equipamiento terminológico en Vietnam. Habiendo destacado una falta de recursos terminográficos relativos a los términos del ámbito de la construcción vietnamita, Trang-Nguyen (2013) realiza un estudio sobre la descripción del vocabulario y el valor de los términos en uso en la práctica terminológica del área. Al hacerlo, asume el enfoque socioterminológico para la elección de los términos y, también, para su análisis en sus relaciones léxicas, lo cual conduce a la producción de un recurso socioterminográfico en línea (on line) para ingenieros civiles. En efecto, en esta obra, el recurso al planteamiento socioterminológico radica en la consideración de los términos desde el punto de vista de su formación, sus significados, su evolución y sus utilizaciones en el discurso profesional.

\subsubsection{Vulgarización de la terminología médica}

La tesis de Alrashidi (2016) aborda la problemática de la difusión social de la terminología médica en Kuwait a través de los vocablos sobre la diabetes. El autor se centra en los datos terminológicos de las obras de divulgación que analiza desde un planteamiento socioterminológico. Tras presentar los problemas socioterminológicos que deben tenerse en cuenta en la vulgarización de la terminología médica, Alrashidi llega a resultados que, a su juicio, ayudarán a manejar mejor las cuestiones que entraña la vulgarización de la terminología médica en el país. 
Este recorrido, que no pretende ser exhaustivo, atestigua la importancia otorgada a lo social de la terminología (o del discurso especializado) en las obras de gran calado que aplican los métodos preconizados por la socioterminología. Se han abordado todos los aspectos (descripción, circulación social, etcétera) y se han tenido en cuenta muchos campos científicos, en varios idiomas (francés, árabe, vietnamita, entre otros). Sin embargo, cabe señalar que idiomas menos lexicalizados en el ámbito científico, especialmente los del África subsahariana, todavía no han sido objeto de estudios socioterminológicos a fondo (Diallo, 2020), a fin de que puedan ser instrumentos eficientes de difusión de los conocimientos especializados. A pesar de ello, se hará especial hincapié en la tesis del autor de estas líneas (Diallo, 2020), quien ha realizado el primer estudio socioterminológico de campo extenso sobre un idioma del África negra.

En su tesis, además de haber aplicado todas las recomendaciones pertinentes del enfoque socioterminológico al estudio del discurso médico en pulaar, Diallo (2020, p. 194) llega a desarrollar un nuevo concepto socioterminológico que denomina intercomprensibilidad. Según este autor, es intercomprensible cualquier denominación especializada que conserve el mismo valor semántico desde su concepción hasta su recepción. La intercomprensibilidad es otro de los criterios socioterminológicos desarrollados por Diallo mediante los cuales los hablantes de la lengua pulaar aceptan o rechazan los términos especializados.

\section{Conclusiones}

En este trabajo, se ha tratado de trazar un panorama sobre el status quaestionis acerca de la socioterminología, en tanto que perspectiva de investigación que está recorriendo camino, pese a que el uso e incluso el éxito de este planteamiento en muchas áreas es limitado. Para ello, primero, hemos creado, un historial de la actividad socioterminológica, profundizando en los factores que han contribuido a la creación y a la evolución del concepto. Segundo, hemos abordado el objeto de la socioterminología, haciendo hincapié en su orientación, finalidad y método de análisis. Por último, se han dado a conocer las perspectivas de distintos estudios científicos que se han suscrito a la socioterminología y los resultados a los que han llegado. 
La socioterminología, como hemos mencionado más arriba, es un planeamiento que nació de los límites percibidos respecto de la terminología dominante de tipo wüsteriano en aquel entonces, sobre todo en Francia. En efecto, la TGT ha sido frecuentemente fuente de críticas por haberse construido sobre ideales mucho más que sobre la descripción de fenómenos reales. Cabré (2002, p. 11) abunda en la misma opinión en los términos siguientes:

Actualmente, la propuesta de Wüster es objeto de revisión por parte de muchos especialistas en terminología, ya que, por su carácter reduccionista e idealista, los especialistas la consideran insuficiente para dar cuenta de las unidades terminológicas en un marco comunicativo plural.

Dicho de manera distinta, mientras adopta un enfoque onomasiológico, la propuesta de Wüster se interesa particularmente por la elaboración de denominaciones, la monosemia, la univocidad, la estandarización, así como la normalización, pero a la vez hace caso omiso de aspectos muy importantes, tales como el funcionamiento social y discursivo de los términos.

La socioterminología, por lo tanto, se fundó y se ha desarrollado en respuesta a una demanda social por lo que respecta al vocabulario o discurso especializado. Dicho de otro modo, se interesa principalmente por los usos sociales de los términos, el estudio de su aparición, circulación e implantación. Esto hace que explote los conocimientos conceptuales y metodológicos de la sociolingüística para aplicarlos al discurso especializado. Desde su aparición hasta hoy en día, muchos estudios se han subscrito a esta orientación. Los primeros estudios, que se desarrollaron a finales del último siglo, trataron de fundamentar la socioterminología a nivel teórico, a través de la gestión social de vocabularios o terminologías. A continuación, a partir de principios de este siglo, se ha tratado de averiguar el carácter operativo tanto de los conceptos elaborados como de los métodos preconizados. Todos ellos han sido puestos en práctica. En efecto, se han abarcado la terminología, la divulgación, el desarrollo y la circulación de vocabularios técnicos y científicos en la sociedad, las condiciones de producción de dichos términos, el análisis de los medios de apropiación de los términos, entre otros, y todos han llegado a los resultados esperados. Sin embargo, podemos deplorar el hecho de que casi todos los estudios teóricos se realizaron partiendo del francés y en lengua francesa. Lo mismo se puede decir de los estudios prácticos. Que sepamos, el primer y único trabajo socioterminológico extenso de 
campo en una lengua del África subsahariana fue realizado recién en 2020. Esto se debe a que las orientaciones socioterminológicas no se han difundido de manera exhaustiva en este continente. Dado el retraso que llevan algunas lenguas del África negra en cuanto al vocabulario científico y técnico, cabría considerar si no se deberían enfocar los estudios desde una óptica socioterminológica para cubrir o tapar las lagunas ya existentes en este ámbito.

\section{Referencias bibliográficas}

Alrashidi, B. (2016). Un problème de terminologie médicale au Koweït: la vulgarisation autour du diabète (Tesis doctoral, Université de Rouen Ruan).

An-Vo, T. A. (2015). Terminologie informatique: approche socioterminologique (Tesis doctoral, Université de Rouen, Ruan).

Barité, M. (2000). Glosario sobre organización y representación del conocimiento: clasificación, indización, terminología. Montevideo: Comisión Sectorial de Investigación Científica y Escuela Universitaria de Bibliotecología y Ciencias, Universidad de la República.

Borbujo, A. S. (2001). Terminología y socioterminología. En E. Real, D. Jiménez, D. Pujante y A. Cortijo (eds.), Écrire, traduire et représenter la fête (pp. 657-664). València: Universitat de València. Recuperado de https://docplayer.es/74243826-Terminologia-y-socioterminologia-arturosantos-borbujo-universidad-de-salamanca.html

Boulanger, J. C. (1995). Présentation: images et parcours de la socioterminologie. Meta, 40(2), pp. 194-205. Recuperado de https://www.erudit.org/fr/revues/meta/1995-v40-n2-meta183/002117ar/

Cabré, M. T. (2002). Terminología y lingüística: la teoría de las puertas [en línea]. Estudios de Lingüística Española (ELIES), 16. Recuperado de http://elies.rediris.es/elies16/Cabre.html

Delavigne, V. (1994). Problèmes d'enquêtes en socioterminologie. Secondes journées internationales de Terminologie, pp. 163-178. Recuperado de https://hal.archives-ouvertes.fr/hal-00924208/document

Delavigne, V. (1995). Approche socioterminologique des discours du nucléaire. Meta, 40(2), pp. 308-319. Recuperado de https://hal.archivesouvertes.fr/hal-00924100 
Delavigne, V. (2001). Les mots du nucléaire. Contribution socioterminologique à une analyse de discours de vulgarisation (Tesis doctoral, Université de Rouen, Ruan).

Diallo, S. (2020). Terminologie et vulgarisation du discours médical en pulaar (Tesis doctoral, Université Gaston Berger, Saint-Louis, Senegal).

Faulstich, E. (1999). Principes formels et fonctionnels de la variation en terminologie. Terminology, 5(1), pp. 93-106.

Felbert, H., Lang, F. y Wersig, G. (1979). Terminologie als angewandte Sprachwissenschaft: Gedenkschrift für Univ. Prof. Dr. Eugen Wüster. Munich, Nueva York, Londres, París: Saur.

Gambier, Y. (1987). Problèmes terminologiques des pluies acides: pour une socio-terminologie. Meta 32(3), pp. 314-320. Recuperado de https://www.erudit.org/fr/revues/meta/1987-v32-n3-meta317/002791ar/

Gambier, Y. (2001). Socioterminologie: une terminologie remise sur ses pieds. Terminogramme (102), pp. 107-118.

Gaudin, F. (1990). La praxématique: un apport pour une théorie du terme? Langues et paraxis, año 1990, pp. 71-82.

Gaudin, F. (2003). Socioterminologie. Une approche sociolinguistique de la terminologie. Bruselas: De Boeck \& Larcier S. A.

Gaudin, F. (2005). La socioterminologie. Langages, 1(157), pp. 80-92. Recuperado de https://www.cairn.info/revue-langages-2005-1-page$\underline{81 . h t m}$

Gentile, A. M. (2007). La circulation sociale du discours de la psychanalyse en langue espagnole: un fonctionnement polynomique? (Tesis doctoral, Université de Rouen, Ruan).

Guespin, L. (1993). Normaliser ou standardiser? Le langage et l'homme, XXVIII(4), pp. 213-222.

Guespin, L. y Marcellesi J. B. (1986). Pour la glottopolitique. Langages, 21(83), pp. 5-34. Recuperado de https://www.persee.fr/doc/lgge_0458726x_1986_num_21_83_2493

Guespin, L. y Laroussi F. (1989). Glottopolitique et standardisation terminologique. La Banque des mots, número especial, pp. 5-21. 
Holzem, M. (1997). Apport des recherches en terminologie à la communauté des sciences (Tesis doctoral, Université de Rouen, Ruan).

Ngwaba, C. (2016). Les termes de la gynécologie obstétrique en igbo: enquête sur un domaine tabou dans une langue sans documents écrits (Tesis doctoral, Université Lumière Lyon 2, Lyon). Recuperado de https://tel.archivesouvertes.fr/tel-01433017/document

Tajo, K. (2013). La terminologie bilingue (Arabe-Français) de la surdité: analyse $d u$ discours textuelle et socioterminologique (Tesis doctoral, Université Paris 3 Sorbonne Nouvelle, París). Recuperado de https://scanr.enseignementsuprecherche.gouv.fr/publication/these2013PA030180

Trân, D. T. (1999). La terminologie médicale vietnamienne (Tesis doctoral, Université de Rouen, Ruan).

Trang-Nguyen, T. H. (2013). Analyse de l'usage du vocabulaire des matériaux de construction et description terminologique vietnamienne-française (Tesis doctoral, Université de Rouen, Ruan).

Vecchi, D. M. (1999). La terminologie en entreprise. Formes d'une singularité lexicale (Tesis doctoral, Université de Paris 13, París).

Wüster, E. (1931). Internationale Sprachnormung in der Technik, besonders in der Elektronik: Die nationale Sprachnormung und ihre Verallgemeinerung. Berlín: Bouvier.

Wüster, E. (1974). Die Allgemeine Terminologielehre: Ein Grenzgebiet zweischen Sprachwissenchaft, Logik, Ontologie, Informatik und den Sachwissenchaften. Linguistics, pp. 61-106.

Nota: El editor Mario Barité aprobó este artículo.

Nota de contribución: Sileymane Diallo elaboró el 100\% del manuscrito.

Corrección de estilo realizada por Raquel Souto en el marco del convenio entre la Tecnicatura universitaria en corrección de estilo (FHCE) y la Facultad de Información y Comunicación (Udelar). 